\title{
SU(3) breaking and the pseudo-scalar spectrum in multi-taste QCD
}

\author{
Michael Creutz $z^{1,3, \star, \star \star}$ \\ ${ }^{1}$ Physics Department, Brookhaven National Lab, Upton, NY 11973, USA
}

\begin{abstract}
Using the Sigma model to explore the lowest order pseudo-scalar spectrum with SU(3) breaking, this talk considers an additional exact "taste" symmetry to mimic species doubling. Rooting replicas of a valid approach such as Wilson fermions reproduces the desired physical spectrum. In contrast, extra symmetries of the rooted staggered approach leave spurious states and a flavor dependent taste multiplicity.
\end{abstract}

\section{Introduction}

Despite over 10 years of controversy, the rooting trick[1] proposed for reducing the taste structure of staggered fermions [2-4] is not valid[5, 6]. The issue is that the exact chiral symmetries inherent in the formulation are incompatible with the well known chiral anomaly.

As the algorithm remains popular and these issues are somewhat subtle, this talk concentrates on two of the more blatant issues that arise when the $S U(3)$ flavor symmetry is broken via unequal quark masses. First, different elements of the usual $S U(3)$ pseudo-scalar octet appear with different taste degeneracies. Second, a large number of spurious pseudo-scalars appear far from any physical particle masses.

Section 2 reviews the standard sigma model picture of the pseudo-scalar mass dependence on the underlying quark masses. Section 3 augments that argument to include the taste degeneracies inherent in the staggered approach. Section 4 briefly discusses how rooting can work with multiple copies, or replicas, of a valid lattice fermion formulation, such as Wilson fermions[7]. Section 5 contrasts that argument with staggered fermions, which are not replicas of equivalent fermions. Here the inherent propagator structure forces the spurious states of the unrooted theory to survive. Finally Section 6 reiterates the two main points mentioned above.

\footnotetext{
^ Acknowledges partial travel support under contract number DE-AC02-98CH10886 with the U.S. Department of Energy. Accordingly, the U.S. Government retains a non-exclusive, royalty-free license to publish or reproduce the published form of this contribution, or allow others to do so, for U.S. Government purposes.

${ }^{\star}$ Speaker, e-mail: mike@ latticeguy.net
} 


\section{Quark masses and the pseudo-scalar spectrum}

Consider three flavor QCD and the usual pseudo-scalar octet

$$
\begin{array}{ccccc} 
& K_{0} & & K_{+} & \\
\pi_{-} & & \pi_{0}, \eta & & \pi_{+} . \\
& & & \bar{K}_{0} &
\end{array}
$$

This section reviews the lowest-order non-linear sigma model prediction for the mass dependence of these particles on the underlying quark masses. For simplicity, ignore the $\eta^{\prime}$ on the grounds that it acquires a large mass through the anomaly.

Begin with the usual picture of spontaneous chiral symmetry breaking giving a quark condensate

$$
\langle\bar{\psi} \psi\rangle=v
$$

where $\psi$ denotes the quark fields. The non-linear sigma model is an effective theory for fluctuations around this expectation value

$$
\bar{\psi}_{L}^{j} \psi_{R}^{k} \sim v \Sigma^{j k}
$$

Here the roman superscripts denote the quark flavors, which run from 1 to 3 , or equivalently lie in $\{u, d, s\}$. Ignoring radial fluctuations, the matrix $\Sigma$ is taken to lie in the group $S U(3)$.

Introduce the eight Gell-Mann matrices $\lambda_{\alpha}$ normalized

$$
\operatorname{Tr} \lambda_{\alpha} \lambda_{\beta}=2 \delta_{\alpha \beta}
$$

Contact with the pseudo-scalar fields follows from

$$
\Sigma=\exp \left(i \pi_{\alpha} \lambda_{\alpha} / f_{\pi}\right)
$$

Here $f_{\pi}$ is a phenomenological constant of about $93 \mathrm{MeV}$.

The kinetic term for the effective field $\Sigma$ takes the form

$$
L_{0}=\frac{f_{\pi}^{2}}{4} \operatorname{Tr}\left(\partial_{\mu} \Sigma^{\dagger} \partial_{\mu} \Sigma\right)
$$

Expanding this to second order in the pion fields gives their effective kinetic term

$$
L_{0}=\text { const }+\frac{1}{2} \partial_{\mu} \pi_{\alpha} \partial_{\mu} \pi_{\alpha}+\ldots
$$

When the quark masses vanish, the starting theory has two chiral symmetries under the rotations

$$
\begin{aligned}
& \psi_{L} \rightarrow \psi_{L} g_{L} \\
& \psi_{R} \rightarrow \psi_{R} g_{R}
\end{aligned}
$$

where $g_{L}$ and $g_{R}$ are independent global elements of $S U(3)$. In the effective theory this symmetry takes the form

$$
\Sigma \rightarrow g_{L}^{\dagger} \Sigma g_{R}
$$

The spontaneous breaking of this symmetry gives the usual octet of pseudo-scalars. 


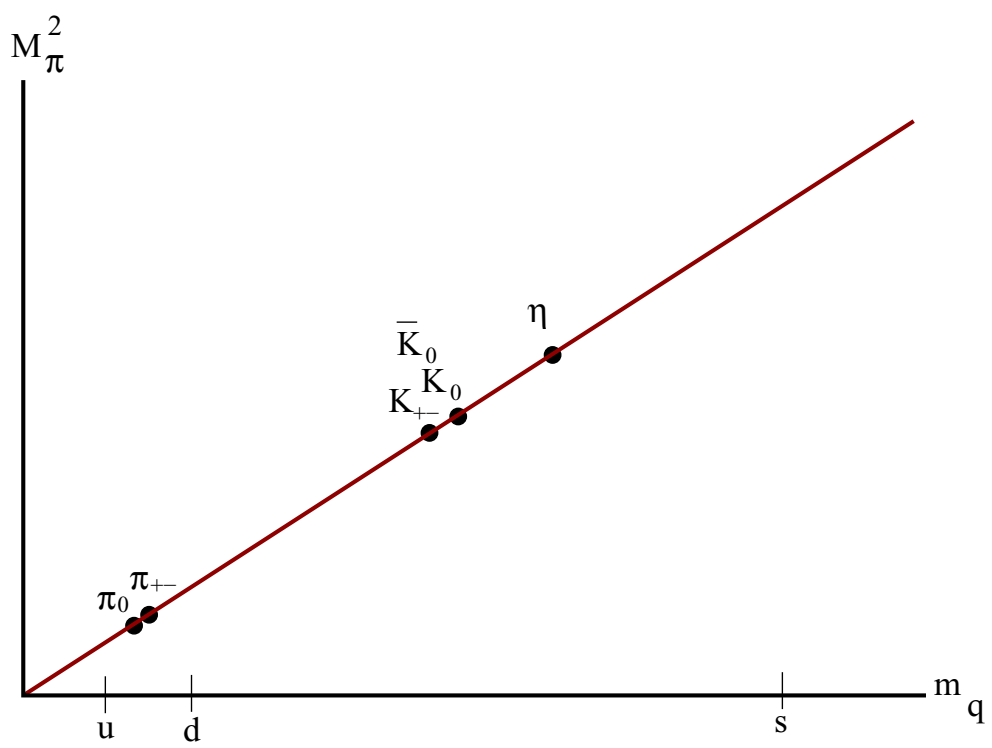

Figure 1. The pseudo-scalar spectrum for fixed quark masses as predicted by the effective sigma model. The quark masses are indicated on the $\mathrm{x}$ axis.

Quark masses break both the flavor $S U(3)$ and the above chiral symmetries. ${ }^{1}$ For the effective theory add a mass term and consider

$$
L=L_{0}-\frac{v f_{\pi}^{2}}{4} \operatorname{Re} \operatorname{Tr}(m \Sigma)
$$

with $m$ a 3 by 3 matrix. Chiral rotations of form $m \rightarrow g_{R}^{\dagger} m g_{L}$ allows $m$ to be put in diagonal form

$$
m=\left(\begin{array}{ccc}
m_{u} & 0 & 0 \\
0 & m_{d} & 0 \\
0 & 0 & m_{s}
\end{array}\right) .
$$

Expanding $L$ to quadratic order in the pion fields gives

$$
L=\text { const }+\frac{1}{2} \partial_{\mu} \pi_{\alpha} \partial_{\mu} \pi_{\alpha}+\frac{1}{2} \pi_{\alpha} M_{\alpha \beta} \pi_{\beta}
$$

where the 8 by 8 meson mass matrix $M$ takes the form

$$
M_{\alpha \beta}=\operatorname{Re} \operatorname{Tr} \lambda_{\alpha} m \lambda_{\beta}
$$

\footnotetext{
${ }^{1}$ Electromagnetic effects are ignored here.
} 
From this it is elementary algebra to obtain the octet masses

$$
\begin{aligned}
& M_{\pi_{+}}^{2}=M_{\pi_{-}}^{2} \propto \frac{m_{u}+m_{d}}{m_{u}^{2}} \\
& M_{K_{+}}^{2}=M_{K_{-}}^{2} \propto \frac{m_{u}}{m_{s}} \\
& M_{K_{0}}^{2}=M_{\bar{K}_{0}}^{2} \propto \frac{m_{d}}{2} \\
& M_{\pi_{0}}^{2} \propto \frac{1}{3}\left(m_{u}+m_{d}+m_{s}-\sqrt{m_{u}^{2}+m_{d}^{2}+m_{s}^{2}-m_{u} m_{d}-m_{u} m_{s}-m_{d} m_{s}}\right) . \\
& M_{\eta}^{2} \propto \frac{1}{3}\left(m_{u}+m_{d}+m_{s}+\sqrt{m_{u}^{2}+m_{d}^{2}+m_{s}^{2}-m_{u} m_{d}-m_{u} m_{s}-m_{d} m_{s}}\right)
\end{aligned}
$$

Note that this involves solving a quadratic equation for the $\pi_{0} \eta$ mixing arising because $M_{38}$ does not vanish. This spectrum is qualitatively sketched in Fig. 1.

\section{Including taste degeneracy}

Motivated by the four tastes inherent with staggered fermions, introduce a factor of $N_{t}=4$ degeneracy for each quark flavor. This leaves us with 12 distinct quark species[8]. To keep the algebra simple, assume that this "taste" symmetry is exact for each of the original three "flavors." Before the breaking of flavor by the quark masses, the chiral symmetry becomes $S U(12) \otimes S U(12)$. Thus there should be $143=12^{2}-1$ pseudo-Goldstone bosons. The 8 by 8 meson mass matrix becomes 143 by 143 , which will now be diagonalized. ${ }^{2}$

This diagonalization is simplified since there are actually three distinct $S U(4)$ taste groups, one for each of the flavors $u, d, s$. This makes it possible to classify the pseudo-scalar mesons in terms of their representations under each of these groups. The relevant $S U(4)$ representations are the $1,4, \overline{4}, 15$ in analogy to the $S U(3)$ representations $1,3, \overline{3}, 8$.

The kaons and charged pions are particularly easy to treat. Each involves two distinct flavors, $q$ and $q^{\prime}$. The mesons appear in the representation $\left(4_{q}, \overline{4}_{\bar{q}^{\prime}}\right)$. Thus they form multiplets of 16 mesons each. The meson masses squared are proportional to the average of their constituent masses, i.e. $M^{2} \propto \frac{1}{2}\left(m_{q}+m_{q^{\prime}}\right)$. With four kaons and two charged pions, this accounts for $6 \times 16=96$ of our total 143 expected pseudo-Goldstone particles.

Now turn to the neutral mesons, those for which a quark is combined with its anti-quark. For this use the breakdown $4 \otimes \overline{4} \rightarrow 1 \oplus 15$. For each flavor, begin with a taste 15 plus a taste singlet.

Remarkably, the taste 15 combinations for each of the flavors cannot mix. This is because each flavor has its own taste group. This gives

- a taste 15 of $i \bar{u} \gamma_{5} u$ states with $M^{2} \propto m_{u}$,

- a taste 15 of $i \bar{d} \gamma_{5} d$ states with $M^{2} \propto m_{d}$,

- a taste 15 of $i \bar{s} \gamma_{5} s$ states with $M^{2} \propto m_{s}$.

None of these has any analogue in the spectrum of Fig. 1.

Finally consider the taste singlet combinations $\bar{u} u, \bar{d} d, \bar{s} s$. From these three, the flavor singlet combination is the $\eta^{\prime}$. As stated earlier, this is heavy and ignored here. The remaining two combinations have the identical mixing matrix as discussed earlier for the single taste theory. These give rise to the masses quoted earlier

$$
\begin{aligned}
& M_{\pi_{0}}^{2} \propto \frac{1}{3}\left(m_{u}+m_{d}+m_{s}-\sqrt{m_{u}^{2}+m_{d}^{2}+m_{s}^{2}-m_{u} m_{d}-m_{u} m_{s}-m_{d} m_{s}}\right) \\
& M_{\eta}^{2} \propto \frac{1}{3}\left(m_{u}+m_{d}+m_{s}+\sqrt{m_{u}^{2}+m_{d}^{2}+m_{s}^{2}-m_{u} m_{d}-m_{u} m_{s}-m_{d} m_{s}}\right) .
\end{aligned}
$$

\footnotetext{
${ }^{2}$ As the goal is an eventual reduction to the normal 3 flavor theory, ignore the possibility of the confining theory reverting to a conformal one.
} 


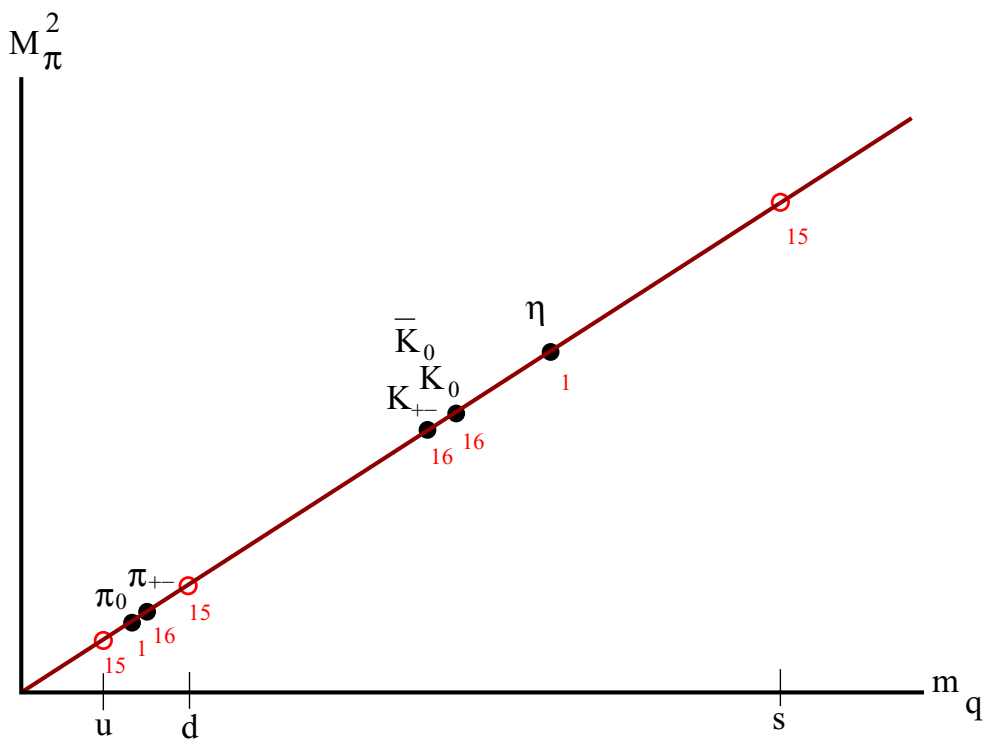

Figure 2. The pseudo-scalar spectrum for the multi-taste theory. The degeneracies of the various states are indicated below the line. The 15-plets, denoted by open circles, do not appear in the single taste theory. Note that the mesons with physical masses do not have a common taste multiplicity.

This relation for the $\eta$ mass in the multi-taste theory appears in Ref. [9].

All anticipated states are now identified, i.e. $143=16 \times 6+15 \times 3+2$. The resulting spectrum is sketched in Fig. 2. The important points to note in comparing this with Fig. 1 are that the taste degeneracies of the physical states depend on which element of the octet one is observing, and there are three multiplets of 15 particles each that have no correspondence in the single taste theory.

\section{Rooting replicas}

Consider a theory with 4 replicas of a valid fermion formulation, such as Wilson fermions. A reduction of the four taste theory down to one taste with the standard rooting trick

$$
|D| \longrightarrow\left|D^{4}\right|^{1 / 4}
$$

is indeed valid. Once the cutoff is in place, $D$ is a finite matrix and this a mathematical identity. ${ }^{3}$ Ignoring doublers, which have been made heavy by the Wilson term, the quark propagator has a single low-energy pole per species. On varying the replica factor $N_{t}$ away from 4 , the state degeneracies evolve as

$$
\begin{aligned}
& 16 \rightarrow N_{t}^{2} \longrightarrow N_{t \rightarrow 1} \quad 1 \\
& 15 \rightarrow N_{t}^{2}-1 \longrightarrow_{N_{t} \rightarrow 1} 0
\end{aligned}
$$

and we obtain the proper spectrum.

\footnotetext{
${ }^{3}$ This technically requires $|D|$ real and non-negative. Interesting cases where this is not true are not considered here.[10]
} 


\section{Staggered fermions}

Now turn to the case of staggered fermions. In this theory, the extra species are not replicas. The doublers all appear in chiral pairs. Whether one roots or not, the propagator always has four light poles. This means that the spurious 15 multiplets will remain in the spectrum even after rooting. Furthermore, the exact chiral symmetry of the staggered approach requires at least one member of each of these taste-15 multiplets to become a Goldstone boson in the chiral limit. Even if we allow for taste breaking, some remnants of the spurious multiplets must remain.

So if the approach is fundamentally flawed, why do previous calculations with this method frequently appear to be fairly accurate? The issues are connected with so called "disconnected diagrams." These are fundamental to the mixing between the strange and the light quarks inherent in the eta meson. Most previous staggered calculations have concentrated on particles dominated by valence quarks, ones that propagate without such direct mixing. For these, the problems are swept into the sea quarks. Such are known to contribute of order ten percent relative to results in the valence[11] or quenched[12] approximation, where the sea is ignored. Furthermore, the sea quark contributions will primarily differ because of incorrect multiplicities in the "pion cloud." In the isospin limit the staggered theory has 63 degenerate pions. This is reduced to $63 / 16$ effective pions after the rooting trick. This compares with the physical cloud composed of of 3 pions. Thus the final error for valence physics is expected to be reduced to a few percent. Larger problems are expected when disconnected diagrams are crucial. This should be particularly serious for the physics of the eta and eta-prime mesons as well as for isospin breaking effects.

\section{Summary}

This discussion raises two issues that practitioners of rooted-staggered fermions should address

1. How can the differing taste multiplicities of pseudo-scalar-octet members be reconciled?

2. How can the three unphysical taste-15 multiplets with unphysical masses be eliminated?

Without answers to these questions, the approach can at best be regarded as an uncontrolled approximation to QCD.

\section{References}

[1] C. Bernard, M. Golterman, Y. Shamir, PoS LAT2006, 205 (2006), hep-lat/0610003

[2] J.B. Kogut, L. Susskind, Phys.Rev. D11, 395 (1975)

[3] L. Susskind, Phys.Rev. D16, 3031 (1977)

[4] H. Sharatchandra, H. Thun, P. Weisz, Nucl.Phys. B192, 205 (1981)

[5] M. Creutz, Phys. Lett. B649, 230 (2007), hep-lat/0701018

[6] M. Creutz, PoS LAT2007, 007 (2007), 0708 . 1295

[7] K.G. Wilson, Erice Lectures 1975 (1977), new Phenomena In Subnuclear Physics. Part A. Proceedings of the First Half of the 1975 International School of Subnuclear Physics, Erice, Sicily, July 11 - August 1, 1975, ed. A. Zichichi, Plenum Press, New York, 1977, p. 69, CLNS-321

[8] W.J. Lee, S.R. Sharpe, Phys. Rev. D60, 114503 (1999), hep-lat/9905023

[9] C. Aubin, C. Bernard, Phys. Rev. D68, 034014 (2003), hep-lat/0304014

[10] M. Creutz, Annals Phys. 339, 560 (2013), 1306.1245

[11] D. Weingarten, D. Petcher, Phys.Lett. B99, 333 (1981)

[12] H. Hamber, G. Parisi, Phys. Rev. Lett. 47, 1792 (1981), [,619(1981)] 